\title{
A 200 AÑOS DE INDEPENDENCIA EN EL PERÚ Y EL PAPEL DE LA HISTORIA CONTADO DESDE LA OTREDAD
}

\section{TO 200 YEARS OF INDEPENDENCE IN PERU AND THE ROLE OF HISTORY COUNTED FROM THE OTREDAD}

\author{
Jesús Wiliam Huanca Arohuanca ${ }^{1 *}$ \\ jhuancaar@unsa.edu.pe \\ ${ }^{1}$ Universidad Nacional de San Agustín de Arequipa, Arequipa, Perú
}

* Correspondencia: Jesús Wiliam Huanca Arohuanca. Email: jhuancaar@unsa.edu.pe

Recibido: 13.07.21 | Aprobado: 08.08.21

\section{RESUMEN}

La idea de República ha colapsado cuando los estudiosos en historia, optaron por la parcialidad al solo resaltar el papel de los personajes principales, dejando de lado a los verdaderos protagonistas de la historia. El constructo se despliega dentro del diseño cualitativo, acompañado de la teoría fundamentada en donde se recoge la ontología y la versión suigéneris de los cuatro "otros" que plantean su posición sobre el rol de la historia. La pretensión del tratado no es desmerecer el aporte de los hombres que a diario se esforzaron por mantener a pie la ciencia llamada historia, pero sí cuestionar de forma crítica su papel, al no mostrar ni presentar los acontecimientos vivenciados desde la otredad. De manera que, si hoy este tema está cambiando, existen todavía ciertas taras de seguir escribiendo desde el escritorio de cristal y la comodidad sin criterio sociohistórico; pues, de seguir así, la historia como tal está condenada a nada más que un pasado de mal aliento.

Palabras Clave: Historia, Independencia, otredad, Bicentenario.

\begin{abstract}
The idea of Republic has collapsed when scholars in history opted for partiality by only highlighting the role of the main characters, leaving aside the true protagonists of history. The construct unfolds within the qualitative design, accompanied by the grounded theory in which the ontology and the suigéneris version of the four "others" that pose their position on the role of history are collected. The intention of the treaty is not to detract from the contribution of the men who daily strived to keep the science called history on its feet, but to critically question its role, by not showing or presenting the events experienced from otherness. So, if today this subject is changing, there are still certain shortcomings of continuing to write from the glass desk and comfort without sociohistorical criteria; for, if it continues like this, history as such is condemned to nothing more than a past of bad breath.
\end{abstract}

Keyword: History, Independence, otherness, Bicentennial. 


\section{INTRODUCCIÓN}

"El Bicentenario no significa nada si no somos incluidos y escuchados". Tener que presentar un bosquejo inductivo desde la partera del sentido común, resulta original para el entendimiento de la historia autónoma, sabiendo que, el actor principal es el hombre, pero no ese hombre que aparece y figura como protagonista en los guiones de la historia rutinal; aquí se habla del "otro" como categoría que representa a los olvidados de la historia. De manera que, lo anterior se traduce en hombres y mujeres que habitan en su generalidad los andes, los cuales, llegado el Bicentenario, también tienen algo que decir. Por ende, no se hablará de peruanos o anti peruanos, se hablará de los sin voces, de los negados y de los silenciados sistemáticamente por los hacedores de la historia que aparece en los libros.

Sin embargo, el hecho de hablar de los "otros", de los avatares de la historia republicana, de la nueva sociabilidad y la función revolucionaria que recorre todos los rincones de la modernidad (Glave, 2015), puede tener repercusiones para la historia consolidada. Ya que, mantener la memoria histórica y el discurso histórico que se expone en las diversas investigaciones (Zapata, 2018) sobre el empoderamiento de indígenas en la zona académica, implica la ruptura con la historia habitual (Huanca-Arohuanca, 2021a; 2021b; 2021c; Huanca-Arohuanca, Asqui, et al., 2021). Es más, la mirada genealógica de relación entre la historia contado desde la otredad y la idea de desarrollo histórico normal, permite apreciar los reclamos de soberanía y autodeterminación de los pueblos (Eslava, 2019).

Por ello, la celebración del Bicentenario de la Independencia en los países que ejemplarmente se sacudieron del yugo colonial, parece un momento oportuno para desafiar y continuar conformando sociedades más inclusivas que puedan, además, integrarse entre sí, a partir de sus narrativas históricas compartidas, en vistas a la construcción de un país más democrático (Cavieres-Fernández et al., 2020; Cubas, 2016). Pero, no se puede olvidar que la Independencia del Perú de 1821 fue una tragedia y no trajo ningún cambio políticosocioeconómico relevante. Es decir; las haciendas de orden gamonal, los esclavos y las minas cambiaron de manos, pero no de espíritu ni de hábitos (Contreras, 2011; HuancaArohuanca \& Pilco, 2021a), sumado a ello, no hubo la denuncia correspondiente de los hacedores de la historia nominal ni mucho menos la bibliografía necesaria para expresar la voz de los invisibilizados. En esa connotación, para Silvia Escanilla (2013), en las últimas décadas se ha escrito demasiado sobre la Independencia del Perú, sobre sus antecedentes y sobre sus protagonistas. Sin embargo, aunque pueda resultar sorprendente, esta profusa bibliografía dice poco sobre la guerra en sí misma y menos se ha reflexionado-debatido sobre el papel del "otro" como actor no expuesto por la historia.

\section{METODOLOGÍA}

El orden metodológico se desenvuelve bajo el paradigma cualitativo, acompañado de la teoría fundamentada, quien hace un recorrido a la ontología de los sujetos de la otredad (Trejos-Gil, 2020) dentro de escenarios turbulentos que le ha tocado vivir al Perú, más todavía en momentos de un Bicentenario fracturado por tracciones tanto internos como externos. Endógenos por la exposición a plena luz de anomalías como: corrupción, 
discriminación, colonialidad, diferencia de clases y exclusiones sistemáticas hacia la otredad. Exógenos por la intromisión de los sistemas imperantes a lo largo de la historia republicana y el ascenso de una pandemia con comorbilidades muy altas (GonzálezJaramillo et al., 2020; Farfán-Cano, 2020; Ríos et al., 2021).

La información se organizó a partir de cuatro relatos obtenidos genuinamente desde los actores de la otredad con cierto nivel para inferir los acontecimientos de la historia como tal. Cabe resaltar que los actores encuentran puntos sensibles en la historia oficial parcializado hacia una sola clase, por lo que se pone de relieve la intencionalidad de un viraje en la historia en su contenido de fondo y forma. En ese sentido, se presentan a los cuatro "otros" que hablan sobre el rol jugado por la historia.

\section{Figura 1}

Cuando los "otros" hablan sobre el rol de la bistoria.

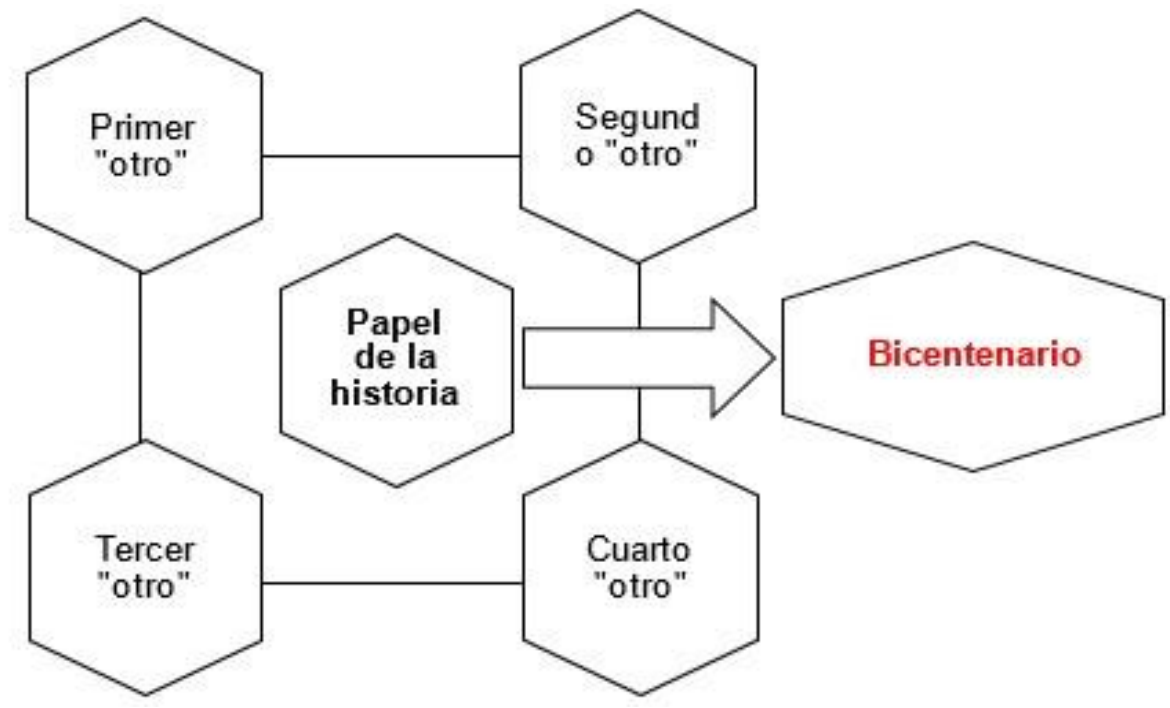

Fuente: elaboración propia.

La ejecución fue sistemáticamente alojada en el constructo, poniendo en primer plano el papel de la historia hasta la entrada del Bicentenario, pero manteniendo el orden cronológico de 200 años de funcionamiento desde la Independencia. En segundo plano, se analizó e interpretó el relato de los cuatro "otros" en función al rol que ha tenido la historia para las civilizaciones peruanas. En tercer plano, se redactó la información obtenida de los actores clave, dejando de lado ciertos enunciados que no correspondían al plenario discutido.

\section{DESARROLLO}

\section{La historia contada desde la óptica de la otredad}

La idea del constructo teórico con pretensiones históricas, resulta válido para dar representatividad y la proposición de la real consecución de los “otros". Porque la vivencia y el entendimiento de la historia, no es lo mismo para los que se sedentarizaron en los rincones del país incaico a diferencia de los que si tuvieron el acceso a la historiografía a través de ciertas universidades y bibliotecas. Pareciera entonces que existió una carencia de autoridad a nivel académico o simplemente primó la negación a la inserción de los 
actores locales al conocimiento de los hechos o quizás no hubo un registro oficial de la otredad.

Aquí el problema es la intencionalidad de dar registro o no a los "otros", porque al final es la intencionalidad del centralismo sobre todo académico, quien justificará ese vació. Como es de conocimiento nacional, la clase indígena agonizó de manera fáctica y en cierta medida sufrió de modo deleznable hasta la Reforma Agraria. Solo con el presidente Juan Velazco Alvarado tuvieron cierta representatividad y reconocimiento. Al respecto, el primer "otro" señala:

¡Acaso nosotros aparecemos en la historia, acaso siquiera nos mencionan!... si existe historia es por nosotros o nuestros antepasados que murieron por ejemplo luchando por la Independencia del Perú [...] No hubo antes y después que Velazco un reconocimiento hacia nosotros [...] me gustaría preguntarles siempre a los historiadores, si lo que estoy hablando será inscrito en la historia o no...

A pesar de ser un tanto provocador lo exclamado por uno de los actores clave de la historia, la obligación de todo historiador sea extranjero o nacional, es recoger y darle sentido a todas las narrativas que están como huellas. Incluso se puede hablar de cuerpos indígenas o de color diseminados por todo el Perú, país que hasta la actualidad no encuentra ciertos grados de integración e inclusión para con sus habitantes.

Al respecto, queda claro que el deber de la historia a partir del Bicentenario desarticulado, se estima en la búsqueda de voces relegadas y la afirmación de la memoria colectiva. Teniendo en cuenta que, la historia no es solo contar de manera tradicional por ejemplo los sucesos beligerantes de una guerra entre posturas de antiguo régimen realista frente a la ideología de modernidad patriótica, sino que es, el de comprender las complejidades de las relaciones de poder de uno y otro bando (Soux, 2010), pero desde una perspectiva que resalte al "otro" y sus múltiples formas de vida manifestadas en su postura ontológica con cierto vínculo tradicional. Al respecto, el segundo "otro" manifiesta:

Estoy seguro que faltó escuchar a los indígenas y los afroamericanos en todo el proceso republicano [...] aunque resulte difícil agregar como pilares de la Historia a estos hombres, la historia hegemónica no logra su integración y menos resaltará las acciones heroicas del indio ante acontecimientos como la Independencia, la Guerra con Chile y otros acontecimientos.

De lo anterior, se extrae la existencia de una matriz colonial en la historia, es decir, existe la creencia de que nadie más que la ralea hegemónica puede hacer y escribir la historia. En consecuencia, con 200 años de historia republicana el papel del mismo como ciencia, solo ha consolidado tanto en la práctica y en la teoría, una sola forma de ver los hechos concretos, pensando que los que son constantemente subalternizados no tienen derecho a hablar. Luego, se tiene la historia que se tiene, una historia negativamente clasificada por los que en su mayoría no han visto de cerca los acontecimientos fácticos ni han observado quiénes realmente son los forjadores de la historia.

Por ahí que la sintonía y el análisis de las guerras de Independencia, las guerras civiles y golpes de Estado de las primeras décadas del siglo XIX (Lossio, 2021) e incluso 
el XX, son solo historias de los vencedores. Lo que sí es evidente, es que los españoles lograron silenciar con la complicidad de una historia clasista a poner en segundo plano, sucesos como la rebelión de Tupac Amaru II, considerándolos como muy peligroso (Walker, 2015) y desafiante hacia el sistema patronal. Por tanto, los puntos de subjetivación y liquidez en la República sobre el manejo de la historia normal, no fue meritorio en sentido estricto y quizás fue un proceso fallido.

Para terminar la idea, en el Perú actual se habla con frecuencia de la República errada que dio como resultado un Bicentenario asaltado por la corrupción y la pandemia mundial, nublando el conjunto de posibilidades para los pueblos subalternos y todo el conjunto de otredades que se presentan hasta hoy. Por lo que, solo volver a la cuestión umbral de los pueblos originarios con historia, hará que mejore las dicotomías existentes, los fenómenos sociales negativos, el silencio al que los pueblos originarios están sometidos (HuancaArohuanca, 2020; Huanca-Arohuanca \& Pilco, 2021b; Huanca-Arohuanca, Sapana, et al., 2021).

\section{¿Quién escribe la historia y para qué?}

En Perú como en otras partes, la "historia es manejada y cocinada desde el escritorio de lujosas comodidades", sumado a ello están las grandes bibliotecas con materiales y archivos. Lo a priori desde luego no está mal, pero sí presenta serias debilidades con lo que debería y no debería ser considerado para que tenga la certeza de ser historia. Si la razón de los peruanos no posee grietas, la historia debe ser escrito desde la acción misma, desde la vivencia real y empírica. En consecuencia, la historia debe ser vivida y sentida por el historiador desde el ángulo que le lleva a escribir.

En esa lógica, para el tercer "otro" la historia debe ser escrita desde los personajes secundarios que en realidad son principales, es más, la historia debe representar más a menudo la visión de los perdedores y no como hasta ahora acostumbra hacerlo.

En estos tiempos, toda la gente habla de San Martín y Bolivar... pero ¿qué hay de la masa que luchó en el campo de batalla, ¿qué hay de los que murieron luchando por la Independencia, que hay de las mujeres que entregaron su vida al servicio de la patria, ¿qué hay de los indígenas que empuñaron el fusil y la onda? La historia nacional solo cuenta lo que al historiador le indican que cuente y deja en la amnesia a los verdaderos héroes.

Sin lugar a dudas, desde la otredad existe un pesimismo enorme sobre el papel jugado por el curioso de la historia, pesimismo que en cierta medida se justifica. Así mismo, hay que resaltar el reclamo que presentan los actores de la historia con la finalidad de ser escuchados y a la par ser registrados como tal. Aunque ahora, con el surgimiento de intelectuales locales con trascendencia, el historiador se ha vuelto más consciente de la realidad, pero en los inicios de la República no lo era. Tanto así que, cumplían y obedecían ciertos intereses de la clase en dominio, lo cual llevó al silencio vorágine a los vencidos y a la orfandad en representación histórica.

Por otra parte, es muy notorio el sesgo y la falta de identidad con la historiografía local, muchos historiadores reconocidos no resaltan ni estudian hechos locales, por el 
simple hecho de que existe la creencia de que con esos temas no lograrán trascender en este mundo académico. Ahí entra a tallar la premisa del cuarto "otro":

Cuando un k'ara vino a la comunidad, buscaba a alguien que entendiera castellano y de preferencia debía ser una autoridad... nunca se acercó a nosotros los que queríamos decir algo. Cuando alguien le preguntó del porque solo hablaba con las autoridades, él respondió: ustedes no tienen nada importante que decir, es más, no creo que puedan decirme algo sobre lo que quiero saber, prefiero buscar en algún archivo local.

Aquí queda expuesto el hombre de historia con todas sus particularidades negativas, es más, se puede inducir que la discriminación del curioso de la historia hacia los "otros" superó todos los niveles. Siendo una dinámica habitual manejada por la mayoría de historiadores en los 200 años de vida republicana, salvo algunas excepciones que sí dieron la talla de un verdadero investigador en historia. Dentro de esa dinámica la respuesta por ese papel del historiador obedece claramente a una estructura económica que habita el mismo, en otras palabras, depende desde dónde y para quién hace investigación en historia.

Entonces, ahora sí se puede responder a la pregunta del quién escribe la historia y para qué. En cuanto a quién; se puede argumentar que es el hombre que nunca sale de su cómoda biblioteca y que por esa misma situación desconoce la realidad; y sobre el para qué, la respuesta puede manejarse desde el pasado, cuando la historia solo era para dar relevancia a la clase privilegiada, mientras que en la actualidad, los historiadores con contenidos locales han ido superando esas deficiencias con una ecuanimidad crítica y con cierta peculiaridad de sentido imperativo.

\section{CONCLUSIÓN}

Ya han pasado 200 años desde la Independencia, y todavía las secuelas quedan como óxido en el fondo de un país que intentó superar los problemas como pudo, pero la resistencia de una clase patriarcal evitó en cada momento los cambios en resguardo total de sus intereses. Sumado a ello, se presentaron una y otra vez las banalidades y las debilidades de fondo y forma para construir una República democrática y sobre todo libre en sentido superestructural.

En definitiva, el papel de la historia representado por sus actores principales, los hombres que escriben la historia, abandonaron sistemáticamente su papel y consecuentemente la vOz de los vencidos, ya que estos fueron arrojados a la oscuridad y al abandono absoluto. En esa composición, se debe tener en cuenta que ahora, los historiadores tienen el protagonismo y la obligación de recoger-escuchar la memoria colectiva de la otredad, de lo contrario pasarán otros 100 años y seguiremos con ese mismo problema.

\section{ORCID}

Jesús Wiliam Huanca Arohuanca: Universidad Nacional de San Agustín de Arequipa, Arequipa, Perú in 


\section{FUENTE DE FINANCIAMIENTO}

El estudio fue autofinanciado.

\section{CONFLICTOS DE INTERÉS}

El autor declara que no existe conflicto de intereses.

\section{AGRADECIMIENTO}

No aplica.

\section{PROCESO DE REVISIÓN}

Este estudio ha sido revisado por pares externos en modalidad de doble ciego.

\section{DECLARACIÓN DE DISPONIBILIDAD DE DATOS}

No aplica.

\section{REFERENCIAS BIBLIOGRÁFICAS}

Cavieres-Fernández, E., Uzcátegui, R., \& Castro, L. (2020). Textos escolares chilenos y peruanos y sus narrativas sobre participación ciudadana durante las independencias nacionales. Implicancias para la formación ciudadana. Diálogo Andino, 63, 271283. https://doi.org/10.4067/s0719-26812020000300271

Contreras, C. (2011, October 7). Independencia sí, Revolución no. Laicacota. http://laicacota.blogspot.com/2011/10/peru-independencia-y-economia-18101825.html

Cubas, R. (2016). Oportunidades de Diversificación Industrial a partir de la demanda de la minería. In Metas del Perú al Bicentenario. Consorcio de Universidades.

Escanilla, S. (2013). La transformación política de la sociedad virreinal. La guerra de independencia en el Perú 1820-1824. XIV Jornadas Interescuelas/Departamentos de Historia. Departamento de Historia de la Facultad de Filosofía y Letras. Universidad Nacional de Cuyo.

Eslava, L. (2019). El estado desarrollista: independencia, dependencia y la historia del Sur. Revista Derecho Del Estado, 43, 25-65. https://doi.org/10.18601/01229893. n 43.03

Farfán-Cano, G. G. (2020). Perspectiva acerca de la enfermedad por coronavirus 2019 (COVID-19). Revista Científica Digital INSPILIP, 4(2), 1-25. https://doi.org/10.31790/inspilip.v4i2.93.g168

Glave, L. M. (2015). Guerra, política y cultura en la génesis de la independencia andina 1808-1815. In C. Contreras \& L. M. Glave (Eds.), La Independencia del Perú: ¿Concedida, conseguida, concebida? (Primera Ed, pp. 305-354). IEP.

González-Jaramillo, V., González-Jaramillo, N., Gómez-Restrepo, C., Palacio-Acosta, C. A., Gómez-López, A., \& Franco, O. H. (2020). Proyecciones de impacto de la pandemia COVID-19 en la población colombiana, según medidas de mitigación. Datos preliminares de modelos epidemiológicos para el periodo del 18 de marzo al 
18 de abril de 2020. Rev. Salud Pública, 22, 1-6. https://doi.org/10.15446/rsap. V22.85789

Huanca-Arohuanca, J. W. (2020). Caleidoscopio social al Covid-19: pánico y desesperación en tiempos de aislamiento. Revista Universidad y Sociedad, 12(6), 226-231. https://rus.ucf.edu.cu/index.php/rus/article/view/1836

Huanca-Arohuanca, J. W. (2021a). Narrativas de guerra y resistencia: participación de la mujer austral del Perú en la Guerra del Pacífico. Encuentros. Revista de Ciencias Humanas, Teoría Social y Pensamiento Crítico, 13, 50-59. https://doi.org/10.5281/zenodo.4395218

Huanca-Arohuanca, J. W. (2021b). Poder mediático y terrorismo de derecha: cuando salvar al Perú es lo único que queda. Revista Revoluciones, 3(4), 1-5. https://doi.org/10.35622/j.rr.2021.04.001

Huanca-Arohuanca, J. W. (2021c). Un fantasma recorre el Perú del Bicentenario: lecciones y voluntad popular en las elecciones de 2021. Revista Revoluciones, 3(3), 1-4. https:// doi.org/10.35622/j.rr.2021.03.001

Huanca-Arohuanca, J. W., Asqui, M. L., Mamani, D., Mamani-Coaquira, H., Huayanca, P. C., \& Charaja, F. (2021). Habilidades lingüísticas y comprensión lectora en la oquedad del siglo XXI: una mirada a la Institución Educativa Politécnica de Puno - Perú. Horizontes. Revista de Investigación En Ciencias de La Educación, 5(18), 537-555. https://doi.org/10.33996/revistahorizontes.v5i18.194

Huanca-Arohuanca, J. W., \& Pilco, N. (2021a). Acciones revolucionarias en Ámérica Latina: Puno y el Alto Perú durante el proceso de independencia (1809-1825). Chakiñan. Revista de Ciencias Sociales y Humanidades, 14. https://doi.org/10.1590/ SciELOPreprints.1364

Huanca-Arohuanca, J. W., \& Pilco, N. (2021b). Transición del virreinato a la República: caleidoscopio sociopolítico-económico del altiplano puneño en la Independencia de Perú (1815-1825). Diálogo Andino, 65, 379-391. http://dialogoandino.cl/ wpcontent/uploads/2021/07/28-HUANCA-PILCO-RDA-65.pdf

Huanca-Arohuanca, J. W., Sapana, N., Casa, M. D., \& Vargas, K. R. (2021). Articulación entre pedagogía y las relaciones humanas: un análisis a los estudiantes quechuas del Instituto Horacio Zevallos Gámez - Cusco. Horizonte de La Ciencia, 11(20), 216226. https://doi.org/10.26490/uncp.horizonteciencia.2021.21.907

Lossio, J. (2021). Pandemias y salud pública. Historias de cuarentenas y vacunaciones (1.a ed). Ministerio de Cultura del Perú.

Ríos, P., Calderón, L., Piedra, S., Guerrero, J., \& Narváez, A. (2021). Proposal for territorial analysis to face the SARS-CoV-2 pandemic based on the Profile of pneumonia and Influenza in Ecuador 2016-2018. Atencion Primaria, 53(5), 102021. https://doi.org/10.1016/j.aprim.2021.102021 
Soux, M. L. (2010). El complejo proceso hacia la independencia de Charcas (1808-1826). Guerra, ciudadanía, conflictos locales y participación indígena en Oruro. Institut français d'études andines. https://doi.org/10.4000/books.ifea.6308

Trejos-Gil, C. A. (2020). Implicaciones ontológicas en el aprendizaje a partir del distanciamiento social impuesto por la pandemia COVID-19. Utopía y Praxis Latinoamericana, 25, 231-247. https://doi.org/10.5281/zenodo.4087453

Walker, C. (2015). Tupac Amaru y el bicentenario. Revista Argumentos, 3, 25-29. https://argumentos-historico.iep.org.pe/articulos/tupac-amaru-y-el-bicentenario2/

Zapata, M. (2018). A propósito del bicentenario: Ambigüedades en la independencia de Arequipa About the bicentennial: Ambiguities in the independence of Arequipa. Revista de Investigación Científica UNTRM: Ciencias Sociales y Humanidades, 1(1), 64-75. https://doi.org/10.25127/rcsh.20181.285

Huanca Arohuanca, J. W. (2022). A 200 años de Independencia en el Perú y el papel de la Historia contado desde la otredad. Puriq, 4, e216. https://doi.org/10.37073/puriq.4.1.216 